\title{
Impact of semaphorin expression on prognostic characteristics in breast cancer
}

This article was published in the following Dove Press journal:

Breast Cancer - Targets and Therapy

\section{Ramesh Butti \\ Totakura VS Kumar \\ Ramakrishna Nimma \\ Gopal C Kundu}

Laboratory of Tumor Biology, Angiogenesis and Nanomedicine Research, National Centre for Cell Science, Savitribai Phule Pune University, Pune, India
Correspondence: Gopal C Kundu Laboratory of Tumor Biology, Angiogenesis and Nanomedicine Research, National Centre for Cell Science (NCCS), Pune 4I I007, India Tel +9l 2025708104 Email kundu@nccs.res.in.

\begin{abstract}
Breast cancer is one of the major causes of cancer-related deaths among women worldwide. Aberrant regulation of various growth factors, cytokines, and other proteins and their receptors in cancer cells drives the activation of various oncogenic signaling pathways that lead to cancer progression. Semaphorins are a class of proteins which are differentially expressed in various types of cancer including breast cancer. Earlier, these proteins were known to have a major function in the nerve cell adhesion, migration, and development of the central nervous system. However, their role in the regulation of several aspects of tumor progression has eventually emerged. There are over 30 genes encoding the semaphorins, which are divided into eight subclasses. It has been reported that some members of semaphorin classes are antiangiogenic and antimetastatic in nature, whereas others act as proangiogenic and prometastatic genes. Because of their differential expression and role in angiogenesis and metastasis, semaphorins emerged as one of the important prognostic factors for appraising breast cancer progression.

Keywords: breast cancer, tumor microenvironment, semaphorins, plexins, neuropilins, cancer stem-like cells, prognostic factor, angiogenesis, metastasis, epithelial to mesenchymal transition, vascular endothelial growth factor
\end{abstract}

\section{Introduction}

Breast cancer accounts for one of the largest causes of morbidity and mortality among women globally. ${ }^{1}$ Intricate signaling pathways regulated by an array of growth factors, cytokines and other proteins are known to be involved in the progression of the incipient neoplasm to higher grades of breast tumors. ${ }^{2}$ Advanced stages of breast cancer are mostly untreatable due to its aggressive nature and lack of effective therapies for the heterogeneous disease. It takes several measures toward identifying the diagnostic and prognostic factors and methods for detection and prevention of cancer at early stages of the disease. Differentially expressed genes/proteins between cancer and adjacent normal/healthy breast tissues are routinely used for the diagnosis and prognosis of breast cancer. Proteomic and microarray studies have identified various genes that are differentially expressed among normal and different grades of breast cancer. ${ }^{3,4}$ Semaphorins are a class of one such proteins that are differentially expressed in normal vs. different grades of breast cancer patients. ${ }^{5,6}$ Semaphorins are a class of secreted, membrane-bound, or glycophosphatidylinositol-anchored glycoproteins and are characterized by the presence of a sema and plexin-semaphorin integrin (PSI) domains. Semaphorins were originally discovered as axon guidance cues for the developing nervous system. ${ }^{7}$ However, the role of semaphorins in regulation of several hallmarks 
of cancer has been eventually recognized. Semaphorins exert their tumor modulatory function by binding to receptors, plexins, and neuropilins, or holoreceptor complexes associated with plexins/neuropilins such as integrins and receptor tyrosine kinases (RTKs) such as C-Met and ErbB2. By binding to these receptors, semaphorins regulate various downstream signaling molecules such as extracellular signal-regulated kinases1/2 (ERK1/2), Akt, phosphatase and tensin homolog (PTEN), and Rho-associated protein kinase, leading to cancer cell survival, angiogenesis, and metastasis. ${ }^{8-10}$ Mishra et al have shown that Sema3A inhibits the tumor growth and angiogenesis by inducing MelCAM expression. ${ }^{11}$ Several reports suggest that semaphorins regulate angiogenesis and metastasis by competing with vascular endothelial growth factor (VEGF) family members for neuropilin binding. ${ }^{12-14}$ Semaphorins also induce the epithelial to mesenchymal transition (EMT) to increase the migratory and invasive potentials of breast cancer cells. ${ }^{15}$ Differences in semaphorin expression can be used clinically to predict the breast cancer subtype, disease progression, and patient's survival. ${ }^{6,16-18}$ Based on the clinical relevance of semaphorin expression and its critical role in disease progression, semaphorins emerged as one of the intriguing therapeutic targets for breast cancer management. In this review, we summarize the clinical relevance of semaphorin expression and the proand antiangiogenic and metastatic effects of semaphorins in breast cancer progression.

\section{General structure and classification of semaphorins}

Twenty different types of semaphorins in humans, five in Drosophila, and two in DNA viruses have been identified. To simplify the understanding of the biology of these semaphorins, semaphorins have been categorized into eight subclasses on the basis of their structural elements and amino acid sequence homology. Out of the eight subclasses, class 1 and 2 semaphorins are present in invertebrates; classes 3 , 4,6 , and 7 semaphorins are only expressed in vertebrates; and the eighth group (class $\mathrm{V}$, where $\mathrm{V}$ stands for the virus) contains semaphorins that are encoded by viral genomes (Figure 1). However, class 5 semaphorins are expressed in both invertebrates and vertebrates. In the current unified nomenclature of semaphorins, the abbreviation Sema is followed by a number indicating the subclass and a capital letter designating the individual member (eg, Sema3A). ${ }^{19}$ All semaphorins possess a conserved sema domain, consisting of 500 amino acids at the $N$-terminal region (Figure 1). ${ }^{20}$ The sema domain constitutes distinctive structural and functional element of semaphorins and is responsible for various functions..$^{21}$ Interestingly, the sema domain is also present in some of the semaphorin receptors such as plexins and RTKs such as MET and RON (Figure 1). ${ }^{22,23}$ Semaphorin, MET, and RON axes are known to play a major role in the development, tissue regeneration, and carcinogenesis. ${ }^{24,25}$ The structure of the sema domain is a seven-blade $\beta$-propeller fold that shows complete structural similarity to the extracellular domain of $\alpha$-integrin. ${ }^{20,25}$ Nevertheless, studies on crystal structures suggest that the mode of dimerization and the regions of the domain involved in ligand-receptor interactions are considerably different among these families. ${ }^{23}$ Next to the sema domain, semaphorins contain a cysteine-rich PSI domain, which is also referred as a MET-related sequence (Figure 1). ${ }^{26}$ Semaphorins also harbor other distinctive protein domains such as basic charged $C$-terminal domain, thrombospondin repeats, and immunoglobulin (Ig)-like domains. Class 3 semaphorins are characterized by a conserved, basic charged domain at the $C$-terminal region and these are secreted semaphorins (Figure 1). ${ }^{10}$ Class 4-7 semaphorins are cell membrane-anchored proteins that are characterized by their distinct structural elements. Thrombospondin repeats are present in case of class 5 semaphorins, whereas a glycophosphatidylinositol anchor is present in class 7 semaphorins (Figure 1). Membrane-anchored semaphorins can be further processed into soluble forms through the proteolytic cleavage at a specific site as in the case of class 4 and 7 semaphorins by ADAMTS1 and furin-like proprotein convertase (FPPC; Figure 1)..$^{27,28}$

Semaphorins bind to plexins and neuropilins to exert their functions through the activation of downstream signaling molecules. ${ }^{29,30}$ Plexins are high-affinity receptors for semaphorins and expressed in both vertebrates and invertebrates. The genome of invertebrates contains two plexin genes, whereas the genome of vertebrates harbors nine plexin genes. Plexins are divided into four subfamilies, which are plexin-A (1-4), plexin-B (1-3), plexin-C1, and plexin-D1 (Figure 1). Plexin contains a sema and PSI domains as similar to their ligands, semaphorins, along with three distinctive Ig-like fold shared by plexin and transcription factors domains. However, plexins lack the homology to any known proteins or functional motifs at the cytoplasmic tail. ${ }^{31}$ The cytoplasmic tail possesses two stretches of amino acids that are rarely similar to GTPase activating proteins (Figure 1). ${ }^{32}$ Neuropilins act as obligate coreceptors for class 3 semaphorins and these are only expressed in vertebrates. Neuropilins are divided into two subclasses, NRP1 and NRP2. Neuropilins, single-pass transmembrane proteins, were initially identified 


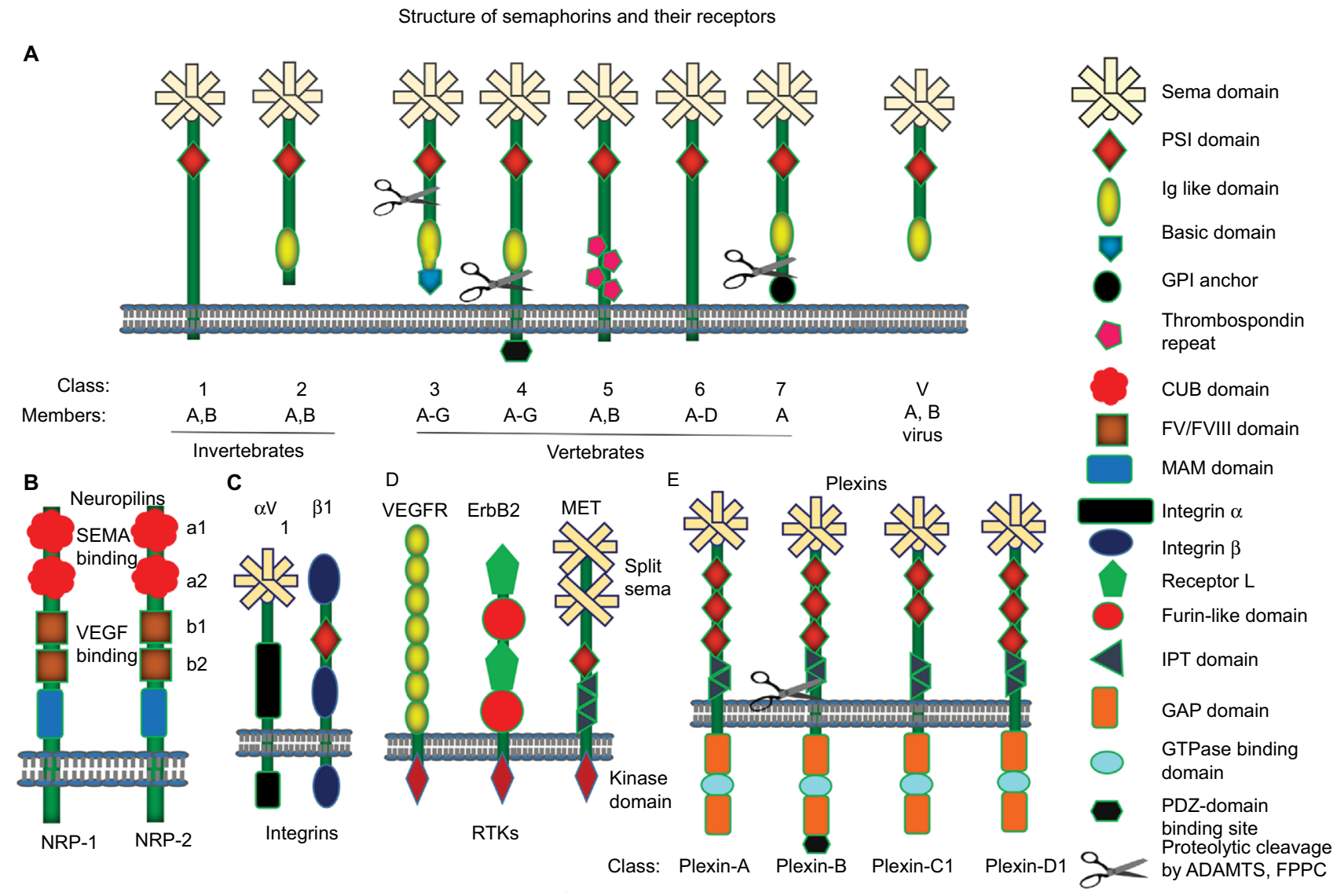

Figure I Graphic representation of semaphorins and their receptors.

Notes: Semaphorins are divided into eight subclasses (classes I-7 and class V). (A) All semaphorins are characterized by a sema domain and PSI domain. Among the vertebrate semaphorins, class 3 is secreted and classes 4, 5, and 6 are membrane bound, whereas class 7 semaphorins are GPI-anchor membrane-bound glycoproteins. Class 3 semaphorins have $C$-terminal basic-charged domain, which is required for binding to neuropilins. Several class 3, 4, and 7 semaphorins undergo controlled proteolytic cleavage by FPPCs or metalloproteases (ADAMTSI). Class 5 semaphorins contain thrombospondin repeats. (B) Neuropilins are single-pass transmembrane receptors that are characterized by the presence of two CUB domains, two FV/FVIII coagulation factor-like domains, and one MAM domain. (C) Integrins $\alpha$ V and $\beta$ I are characterized by the presence of a sema and PSI domains, respectively. (D) VEGFR possesses Ig-like and kinase domain. ErbB2 contains furin and receptor-L domains. (E) Plexins harbor one sema domain, two to three PSI domains and three IPT domains. The cytoplasmic domain of plexin is weekly similar to GAPs. In addition, the B-subfamily plexins contain PDZ domains. A cleavage site for FPPCs also exists in the extracellular domain of plexin-B.

Abbreviations: FPPCs, furin-like proprotein convertase; GAPs, GTP-ase activating proteins; GPI, glycophosphatidylinositol; IPT, Ig-like fold shared by plexin and transcription factors; PSI, plexin-semaphorin integrin; RTKs, receptor tyrosine kinases; VEGFR, vascular endothelial growth factor receptor.

as coreceptors for class 3 semaphorins and VEGF family proteins. ${ }^{33,34}$ Neuropilin comprises two complement-like (CUB) domains and two FV/FVIII coagulation factor-like domains, which are useful for the binding to semaphorins and VEGF family members, respectively. In addition, neuropilins also exhibit meprin-like MAM domain, which is an evolutionarily conserved domain likely to have an adhesive function (Figure 1). ${ }^{35}$ Additionally, semaphorins engage with holoreceptor complexes associated with plexins and neuropilins, such as $\alpha \mathrm{V} \beta$ 1integrin, and RTKs such as Met, ErbB2, and VEGFR2 (Figure 1). ${ }^{28}$

\section{Semaphorin signaling in breast cancer}

Semaphorins regulate several pleiotropic changes that are associated with tumor progression by influencing the behavior of tumor cells. ${ }^{36}$ Except for Sema3E, all other members of class 3 semaphorins bind to plexin-A (1-4) only in the presence of their coreceptors, NRP1 and NRP2. However, they can also directly bind to NRPs to perform their various functions. Sema3E is known to exhibit its tumor-promoting function by binding to plexin-D1.9,30,37,38 Sema4D, another semaphorin, binds to plexin-B1 and B2, whereas Sema4C engages only with plexin-B2..$^{39,40}$ Moreover, Sema5A is known to bind to plexin-B3, while Sema7A is recognized to bind to plexin-C1..$^{30,41}$

Sema3A stimulates the $\alpha 2 \beta 1$ integrin expression, and it ultimately leads to a reduction in the migratory and invasive potential of breast cancer cells. ${ }^{42}$ However, recent studies have shown that Sema3A shows both promoting and inhibitory effects on breast cancer cell migration. Moreover, cell 
migration and spreading are influenced by the strength of cell-substratum interaction. Optimal concentration of substratum leads to increased migration and spreading of cells. Gehler et al have recently demonstrated that Sema3A increases the cancer cell migration and spreading even at a low concentration of the substratum (ECM coating with collagen and fibronectin) by inducing FAK phosphorylation at the focal adhesions in Rho-associated protein kinasedependent manner, while it inhibits the migration at a high concentration of the substratum. ${ }^{43}$ Mishra et al have shown that Sema3A suppresses the breast tumor growth and angiogenesis through PTEN/FOXO 3a axis-dependent MelCAM expression by binding to the receptor, NRP1 (Figure 2). ${ }^{11}$
Sema3A inhibits VEGF-induced activation of ERK1/2 without efficiently disturbing VEGF-induced phosphorylation of VEGFR2 in endothelial cells. ${ }^{44}$ Acevedo et al have revealed that Sema3A suppresses VEGF-induced angiogenesis by interrupting VEGF-mediated FAK and Src signaling in endothelial cells. ${ }^{45}$ Moreover, Sema3A shows a causative role in cancer cell metastasis to the bone by stimulating osteoblastic differentiation. ${ }^{46}$ Another member of class 3 semaphorins, Sema3B, also acts as a tumor suppressor in breast cancer. Sema3B suppresses tumor proliferation and induces apoptosis in NRP1-positive breast cancer cells by inactivating PI3K/Akt signaling (Figure 2). ${ }^{47}$ Sema3B and $\mathrm{VEGF}_{165}$ act as antagonists to each other in the regulation

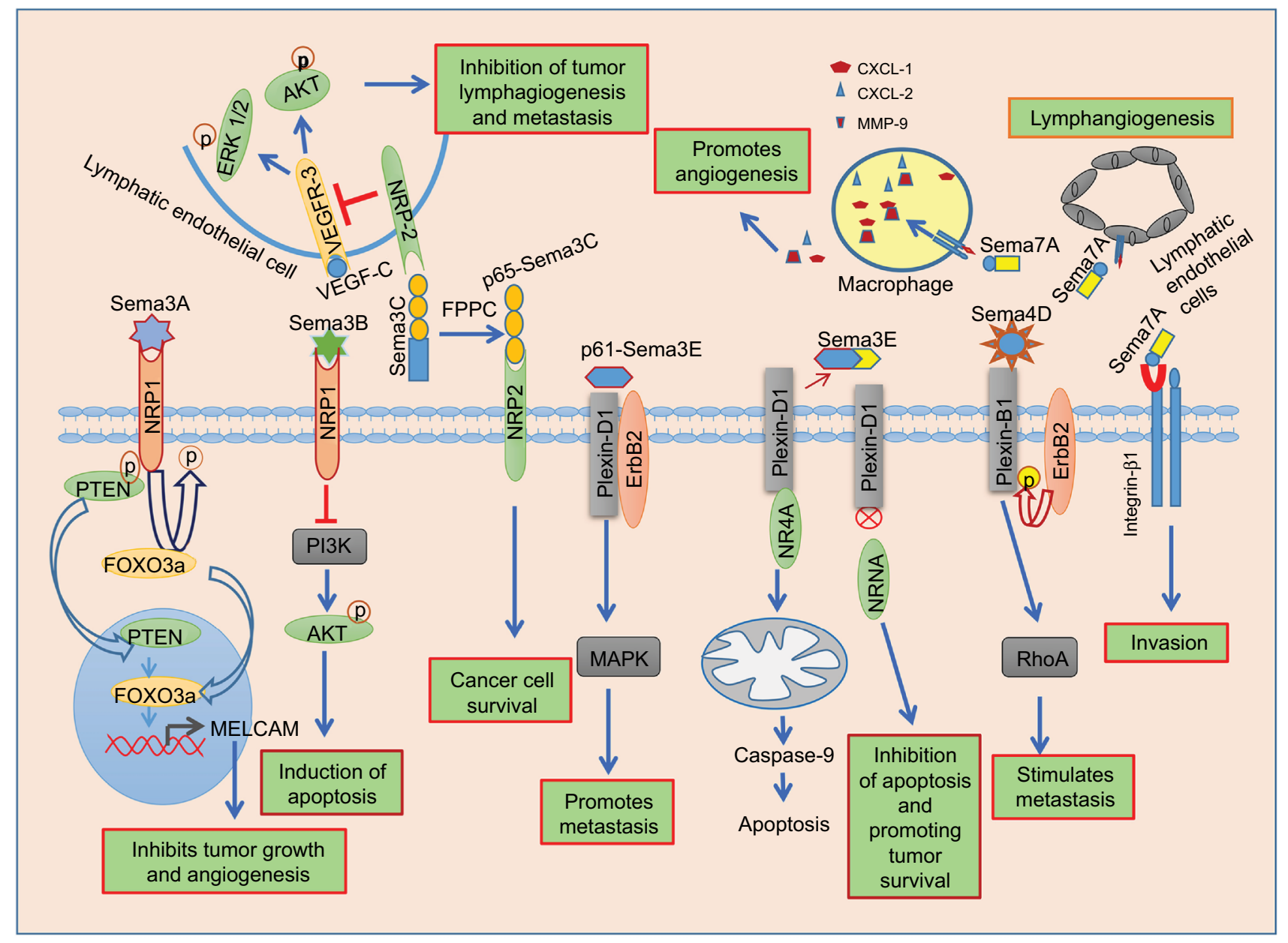

Figure 2 Semaphorin signaling in breast cancer.

Notes: Sema3A interacts with NRPI receptor to induce PTEN/FOXO 3a-dependent MelCAM expression, which, in turn, inhibits tumor growth and angiogenesis. Sema3B binds to NRPI and induces apoptosis by inhibiting PI3K/Akt signaling. Full-length Sema3C interacts with NRP2 on the lymphatic endothelial cells in tumor and suppresses lymphangiogenesis and metastasis by inhibiting VEGF-C-dependent ERKI/2 and Akt signaling. Full-length Sema3C undergoes proteolytic cleavage by FPPC to form p65Sema3C, which promotes cancer cell survival. Sema4D binds to plexin-BI and activates ErbB2, which, in turn, phosphorylates plexin-BI. Phosphorylated plexin-BI induces migration by activating RhoA GTPase. Cleaved p6I-Sema3E binds to plexin-DI to promote metastasis through ErbB2-dependent MAPK signaling. Sema3E binds to plexin-DI to inhibit apoptosis by disrupting the interaction between plexin-DI and NR4A, which is known to induce caspase-9-mediated apoptosis. Sema7A interacts with integrin $\beta$ I on the cancer cells to promote invasion. Tumor-derived Sema7A binds with integrin $\beta I$ on the macrophages to promote angiogenesis by producing CXCL2, CXCLI, and MMP-9.

Abbreviations: ERKI/2, extracellular signal-regulated kinases I/2; FPPC, furin-like proprotein convertase; MMP, matrix metalloproteinase; PTEN, phosphatase and tensin homolog; VEGFR, vascular endothelial growth factor receptor. 
of apoptosis in breast cancer cells. This might be due to the competitive binding of Sema3B and $\mathrm{VEGF}_{165}$ to the NRP1 receptor. ${ }^{14}$ Shahi et al have further shown that Sema3B is a direct transcriptional target of GATA3 and suppresses breast cancer metastasis by interfering with the phosphorylation and activation of LIM kinases (LIMK1 and LIMK2). ${ }^{48}$ The other members of class 3 semaphorins, such as Sema3C and Sema3E, are overexpressed in breast cancer cells and exhibit tumor-promoting function. Zhu et al have shown that siRNA-mediated knockdown of Sema3C in breast cancer cells abolishes cell proliferation and migration. ${ }^{49}$ Interestingly, the p65-Sema3C fragment that is generated from cleavage of full-length Sema3C by FPPC shows tumorpromoting role. Full-length Sema3C shows inhibitory effect on lymphangiogenesis and metastasis in mice breast tumor xenografts (Figure 2). ${ }^{50}$ Nonetheless, the metalloprotease ADAMTS1 induces Sema3C cleavage from ECM and converts it to a soluble form, so that it diffuses and promotes tumor cell migration. ${ }^{51}$ The role of Sema3C in regulation of tumor progression also depends on the type and nature of cancers. For example, Sema3C promotes pancreatic cancer progression through ERK1/2 signaling pathway. ${ }^{52}$ However, the molecular mechanism by which Sema3C promotes breast cancer progression is unclear. Sema3E suppresses the apoptotic cell death in metastatic breast cancer cells by blocking plexin-D1-mediated NR4A1 pathway through binding and sequestering plexin-D1 (Figure 2) ${ }^{53}$ Cleaved p61-Sema3E promotes tumor metastasis through plexin-D1/ErbB2dependent MAPK signaling (Figure 2). ${ }^{38}$ Other members of semaphorin family such as Sema4A, Sema4C, Sema4D, and Sema7A show tumor-promoting function in breast cancer. Sema4C promotes breast cancer cell proliferation and migration through plexin-B2/Met-dependent RhoA signaling axis. In addition, Sema4C induces migration and tube formation of lymphatic endothelial cells (LECs) and, thereby, lymphangiogenesis through plexin-B2/ErbB2-dependent RhoA signaling. ${ }^{54}$ Tumor-derived Sema4D promotes bone metastasis by inhibiting bone deposition and, inducing osteoclastogenesis through the plexin-B1-dependant interleukin-8 secretion. ${ }^{55}$ Effect of Sema4D/plexin-B1 signaling on tumor cell migration depends on their interaction with RTKs, ErbB2, and MET. Interaction of plexin-B1 with MET suppresses cellular migration, whereas interaction with ErbB2 increases the migration by activating the small GTPase, RhoA (Figure 2). ${ }^{56-58}$ Sema7A promotes tumor growth and invasion in breast cancer through activation of integrin $\beta 1$ signaling. ${ }^{59}$ Garcia-Areas et al have observed that cancer cell-derived Sema7A drives the macrophages toward tumor-promoting phenotype, and these macrophages promote angiogenesis by producing proangiogenic molecules such as CXCL1, CXCL2, and matrix metalloproteinase-9 (Figure 2). ${ }^{60}$

\section{Role of semaphorins in breast cancer progression}

Semaphorins regulate various hallmarks of cancer by binding to different types of receptors. Various pathophysiological functions regulated by semaphorins in breast cancer are listed in Table 1.

\section{Role of semaphorins in breast tumor angiogenesis}

Angiogenesis is a process in which sprouting of new blood vessels takes place in order to supply nutrients to rapidly growing tumors. As the tumor grows rapidly, the core part of solid tumor undergoes $\mathrm{O}_{2}$ and nutrient deprivation, a phenomenon known as hypoxia. Hypoxia is the major driving force in inducing tumor angiogenesis by regulating the expression of proangiogenic genes such as VEGF, HIF-1, and so on and enriching cancer stem-like phenotype in the tumor microenvironment. ${ }^{61,62}$ It has also been reported that breast cancer stem-like cells undergo transdifferentiation to endothelial cells to support angiogenesis, which is termed as vasculogenic mimicry. ${ }^{63}$ Various growth factors and cytokines are known to regulate angiogenesis. Semaphorins play crucial roles in angiogenesis directly or indirectly by regulating VEGF/VEGFR axis. ${ }^{12-14}$ Some members of semaphorins, such as Sema4D and Sema7A, are the positive regulators of angiogenesis, whereas the members of class 3 semaphorins are shown to have an antiangiogenic role in breast cancer. 8,60 Especially, Sema3A, Sema3B, Sema3E, and Sema3F exhibit antiangiogenic properties and thereby inhibit tumor progression. ${ }^{8,9}$ It has been reported that class 3 semaphorins inhibit angiogenesis by competing with angiogenic factors, such as members of VEGF family, for binding to neuropilins. ${ }^{12,13}$ Recently, Mishra et al have reported the mechanism by which Sema3A attenuates tumor growth and angiogenesis by inducing the expression of tumor suppressor gene, MelCAM, in breast cancer model. Their studies have shown that Sema3A induces the expression of MelCAM through NRP1-mediated PTEN-dependent FOXO 3a activation. ${ }^{11}$ Casazza et al have shown that overexpression of Sema3A inhibits the vessel formation and increases tumor hypoxia and necrosis in an in vivo mice model ${ }^{64}$ Earlier reports have suggested that cleaved Sema3C (p65-Sema3C) is formed from full-length Sema3C by the action of FPPC. Cleaved Sema3C (p65-Sema3C) is 
Table I Semaphorins, their receptors, and pathologic functions in breast cancer

\begin{tabular}{|c|c|c|c|c|c|}
\hline Class & Members & $\begin{array}{l}\text { Membrane bound } \\
\text { or secreted }\end{array}$ & $\begin{array}{l}\text { Receptors and } \\
\text { coreceptors }\end{array}$ & $\begin{array}{l}\text { Tumor promoting/ } \\
\text { inhibitory }\end{array}$ & Pathologic functions \\
\hline \multirow[t]{11}{*}{ Class 3} & Sema3A & Secreted & NRPI/plexin AI-4 & Inhibitory & Inhibits invasion and migration ${ }^{42}$ \\
\hline & & & & & Suppresses breast tumor growth and angiogenesis.9,11 \\
\hline & Sema3B & Secreted & NRPI/plexin $\mathrm{Al}-4$ & Inhibitory & Inhibits tumor progression and metastasis ${ }^{48}$ \\
\hline & & & $\mathrm{NRP} 2 /$ plexin $\mathrm{Al}-4$ & & \\
\hline & Sema3C & Secreted & $\mathrm{NRPI} /$ plexin $\mathrm{Al}-4$ & Promoting or inhibitory & Inhibits metastasis and lymphangiogenesis ${ }^{50}$ \\
\hline & & & NRP2/plexin Al-4 & & Promotes cell adhesion, proliferation, invasion, and \\
\hline & & & NRPI/plexin DI & & migration ${ }^{6,49}$ \\
\hline & & & NRP2/plexin DI & & \\
\hline & Sema3E & Secreted & Plexin-DI & Tumor promoting & Promotes invasion, migration, and lung metastasis ${ }^{72}$ \\
\hline & Sema3F & Secreted & NRPI & Inhibitory & Inhibits migration ${ }^{70}$ \\
\hline & & & & & Inhibits invasion ${ }^{71}$ \\
\hline \multirow[t]{5}{*}{ Class 4} & Sema4C & Membrane bound & Plexin-B2 & Tumor promoting & Enhances lymphangiogenesis and metastasis ${ }^{54}$ \\
\hline & & & & & Promotes lymphatic metastasis $^{74}$ \\
\hline & & & & & Promotes paclitaxel resistance-induced $\mathrm{EMT}^{76}$ \\
\hline & Sema4D & Membrane bound & Plexin-BI & Tumor promoting & $\begin{array}{l}\text { Promotes proliferation, angiogenesis, migration, invasion, } \\
\text { and antiapoptotic } c^{65,66}\end{array}$ \\
\hline & & & & & Promotes osteoclastogenesis ${ }^{55}$ \\
\hline Class 6 & Sema6D & Membrane bound & Plexin-Al/4 & Inhibitory & Its expression in TNBC is associated with patient survival ${ }^{80}$ \\
\hline \multirow[t]{4}{*}{ Class 7} & Sema7A & GPI-anchor & Plexin-Cl & Tumor promoting & Promotes cell adhesion, proliferation invasion, and \\
\hline & & & Integrins & & lymphangiogenesis ${ }^{59,73}$ \\
\hline & & & & & Promotes EMT ${ }^{15}$ \\
\hline & & & & & Proangiogenic ${ }^{60}$ \\
\hline
\end{tabular}

Abbreviations: EMT, epithelial to mesenchymal transition; GPI, glycophosphatidylinositol; TNBC, triple-negative breast cancer.

required for the survival of NRP2-expressing tumor cells, whereas furin cleavage-resistant Sema3C (FR-Sema3C) is shown to inhibit lymphangiogenesis and metastasis. ${ }^{50}$ ColeHealy et al have found the positive correlation between Sema3C expression and microvessel density (CD31) by immunohistochemical analysis. These studies have shown that the expression of Sema3C is more in endothelial cells of premalignant tissues, suggesting the role of Sema3C in angiogenesis during tumor development. ${ }^{16}$ Jiang et al have revealed that downregulation of Sema4D decreases the tumor growth and angiogenesis. ${ }^{65}$ Tumor-associated macrophages are the major stromal cells that secrete Sema4D in the tumor microenvironment. Tumor-associated macrophage-derived Sema4D contributes to breast cancer angiogenesis and tumor progression. ${ }^{66}$ Another member of semaphorin family, Sema7A, is shown to be upregulated by the proangiogenic molecule, COX-2. Sema7A mediates COX-2-induced lymphangiogenesis by activating $\beta 1$-integrin signaling. ${ }^{59} \mathrm{In}$ addition, Sema7A has been found to induce macrophages to produce proangiogenic molecules such as CXCL2/MIP-2 in an orthotopic breast cancer model ${ }^{60}$ These reports imply the possible role of semaphorins in tumor-stroma interaction in breast cancer.

\section{Role of semaphorins in the invasion and metastasis of breast cancer}

Metastasis is mostly responsible for the cancer-related deaths in different types of cancers. ${ }^{67}$ Cancer cells disseminate to various parts of the body through the blood circulation upon acquiring mesenchymal phenotype by a phenomenon known as EMT. Epithelial cancer cells lack the motility and invasion potentials. Hence, the epithelial cells undergo EMT in order to acquire mesenchymal stem-like phenotype and obtain migration and invasion potentials. ${ }^{68}$ Breast cancer cells are highly metastatic to lungs and bone, depending on the subtype and the hormone receptor status. ${ }^{67}$ Semaphorins are known to play a key role in breast cancer cell dissemination. A recent report has revealed that Sema3C expression is associated with breast cancer cell proliferation and migration. The study has demonstrated that silencing Sema3C using siRNA resulted in suppression of proliferation and migration of estrogen receptor (ER)+ve breast cancer cells, MCF7. ${ }^{49}$ Malik et al have found that Sema3C downregulation reduces the cell adhesiveness and the invasion of human breast cancer cells such as MCF-7 and MDA-MB-231. ${ }^{6}$ It has shown that hypoxia downregulates the expression 
of Sema3A. Hypoxia-regulated Sema3A is known to be involved in the regulation of osteoblast differentiation. ${ }^{69}$ It has been reported that VEGF promotes the migration of cancer cells, whereas Sema3F inhibits this effect owing to the competitive binding of VEGF and Sema3F to the NRP1 $1{ }^{70}$ Retinoid orphan nuclear receptor alpha (ROR $\alpha$ ), a member of the orphan nuclear factor family, inhibits breast cancer cell invasion by enhancing Sema3F expression at the transcriptional level by binding to its promoter. Nuclear levels of ROR $\alpha$ are correlated with Sema3F expression in human breast cancer. Moreover, higher grades of breast cancer are mostly double negative for ROR $\alpha$ and Sema3F, compared to lower grades. Kaplan-Meier log-rank analyses of breast cancer tissue microarray containing $>400$ patients' samples have revealed that patients with lower ROR $\alpha$ and Sema3F have shorter survival rates. ${ }^{71}$ Another member of class 3 semaphorin, Sema3E, is important for tumor progression and metastasis. Mouse mammary carcinoma cell line, 168FARN, gains the ability to metastasize to lungs upon overexpression of Sema3E. Conversion of full-length Sema3E into p61-Sema3E isoform is required for invasion, migration, and lung metastasis. p61-Sema3E isoform is also required for the activation of ERK signaling in endothelial cells. ${ }^{72}$ Garcia-Areas et al have studied the role of Sema7A in tumor growth and metastasis using in vivo mouse model. In this study, they found that downregulation of Sema7A using shRNA decreases the proliferation and reduces the migration and invasion potential of $4 \mathrm{~T} 1$ cells. ${ }^{73}$ Reduction in breast cancer cell adhesion, invasion, and motility was observed upon silencing the expression of Sema7A in MCF10DCIS cells. ${ }^{59}$ Allegra et al have studied the role of Sema7A in regulation of EMT as this process is involved in acquisition of metastatic potential. Their reports have shown that downregulation of Sema7A expression by the Ets2-repressor factor represses the EMT program in Ras-dependent mammary epithelial cells. ${ }^{15}$ These reports indicate that Sema7A increases the migration of breast cancer cells by inducing EMT. Members of Sema4, such as Sema4C and Sema4D, are shown to be positive regulators of metastasis. Chen et al have evaluated the expression of Sema4C in 45 breast tumor specimens and identified higher Sema4C expression in lymph node metastatic specimens as compared to non-metastatic ones. Moreover, they have observed higher expression of Sema4C in metastatic breast cancer cell lines, MDA-MB-231 and MDA-MB-435S, as compared to low-metastatic breast cancer cells, MCF-7. ${ }^{74}$ Secretory Sema4C enhances the migration of breast cancer cells and promotes tube formation and migration of
LECs and thereby enhances the lymphangiogenesis and metastasis in breast cancer. ${ }^{54} \mathrm{Wu}$ et al have isolated the normal LECs and tumor-associated LECs from normal breast and cancer tissues using LCM after detecting these cells in tissue sections by rapid immunohistochemistry. Differentially expressed genes in these cells were analyzed by microarray, and it was found that Sema4C is highly expressed in tumor-associated LECs as compared to normal LECs. ${ }^{75}$ It was shown that miR-125b has an important role in regulating paclitaxel resistance-induced EMT in breast cancer cells by targeting Sema4C. Downregulation of miR-125b and upregulation of Sema4C were observed in paclitaxel-resistant breast cancer cells. ${ }^{76}$ It has been shown that knocking down the expression of Sema4D in MDA-MB-468 and MDA-MB-231 using shRNA reduces the proliferation, invasion, and migration of these cells. Downregulation of Sema4D also increases the apoptosis in these cells. ${ }^{65}$ Furthermore, it has also been observed that Sema4D inhibits bone formation through interaction with plexin-B1 and stimulates osteoclastogenesis through the induction of interleukin- 8 expression and thereby induces bone metastasis of breast cancer cells. ${ }^{55}$ These results suggest the prometastatic role of Sema4D in breast cancer. Evans et al have shown that the expression of Sema4D is higher at the invasive margins of breast tumor where it influences the infiltration of monocytes and leukocytes into tumor microenvironment. Blocking Sema4D using mouse monoclonal antibody, MAb67, leads to tumor rejection in ErbB2+ve murine breast cancer model. Thus, targeting Sema4D in human breast cancer might be beneficial for the treatment of breast cancer. VX15/2503, a humanized monoclonal antibody to Sema4D, is in Phase I clinical trial for the treatment of solid tumors. ${ }^{77}$ Various reports have shown that Sema3B is a tumor suppressor in several cancers including breast cancer. GATA3-induced Sema3B suppresses the breast tumor progression and metastasis by abrogating the phosphorylation and activation of LIMK1 and LIMK2. ${ }^{48}$ Based on the above findings, semaphorins are proved to be crucial targets for the management of metastatic breast cancer.

\section{Clinical relevance of semaphorin expression in breast cancer}

Several reports on clinical studies suggested that semaphorin expression is correlated with disease progression, indicating the prognostic significance of semaphorins in breast cancer. Some members of semaphorin family are downregulated, whereas other members are overexpressed 
during breast cancer progression. It has been reported that the expression levels of Sema3A, Sema3B, and Sema3F are high in normal breast tissues as compared to invasive breast tumors, suggesting the tumor suppressor role of these semaphorins. Similarly, the expression of semaphorin receptor, plexin-A3, is also decreased in invasive breast cancer. ${ }^{5}$ Studies on 119 human breast tumor specimens have revealed that the expression of plexin-B1, a receptor of Sema4D, is inversely correlated with the aggressiveness of this cancer. Moreover, plexin-B1 expression is positively correlated with the ER status of breast cancer. ${ }^{78}$ Microarray dataset of 1086 breast cancer patients has revealed that plexin-B1 has a prognostic value in ER+ve breast cancer. The loss of plexin-B1 is associated with an increased expression of ErbB2 and the proliferation marker, Ki67, in ER+ve breast cancer. In addition, the loss of plexin-B1 expression is associated with poor prognosis in ER+ve breast cancer. ${ }^{79}$ These $^{2}$ reports emphasize the prognostic significance of plexin-B1 in ER+ve breast cancer. In another study, the data revealed that reduced expression of plexin-B1 is associated with poor disease-free survival in ErbB2-ve cancer, whereas in ErbB2overexpressing patients, low plexin-B1 expression levels are associated with high disease-free survival. ${ }^{58} \mathrm{VEGF}$ and semaphorins have been found to exhibit contrasting functions in breast cancer progression. In support of this, metaanalyses of 2656 breast tumor samples have revealed high VEGF and low secreted semaphorin levels in $60 \%$ of total TNBC specimens. Moreover, in non-TNBC patients, higher expression of VEGF and lower expression of semaphorins are associated with low survival rates. ${ }^{17} \mathrm{~A}$ recent report has suggested that reduced levels of Sema4D are associated with poor clinical outcomes and decreased disease-free survival in breast cancer. It was also shown that reduced expression of Sema4D is associated with bone metastasis. ${ }^{18}$ Malik et al have observed that the transcript level of Sema3C is higher in breast cancer tissues as compared to adjacent normal mammary tissues, using real-time polymerase chain reaction analysis of 106 breast tumors and 28 adjacent normal breast specimens. ${ }^{6}$ The data have shown that triple-negative breast tumors have higher Sema3C levels as compared to ER+ve breast tumors, using tissue microarray analysis of 343 breast tumor samples. The data also suggested that Sema3C expression is high in ER-ve, PR-ve, and HER2+ve breast tumors. ${ }^{16}$ Expression of Sema6D in human breast cancer was analyzed using the human cancer genome atlas database, and it was found that the expression of Sema6D was correlated with overall survival in TNBC patients, and this might be helpful in predicting the progression of this subtype of breast cancer. ${ }^{80}$ These studies suggest that semaphorins and their receptors are promising prognostic factors for the prediction of breast cancer progression.

\section{Conclusion}

Some of the members of the semaphorin family exhibit antitumorigenic effect, whereas others show protumorigenic effect in breast cancer by regulating tumor growth, angiogenesis, and metastasis. Semaphorin expression is dysregulated during tumor progression. Differential expression of semaphorins is associated with the disease progression of different subtypes of breast cancer. Higher expression levels of Sema3C and Sema3E are associated with increased tumor aggressiveness and poor prognosis in breast cancer patients. Lower expression levels of Sema3A, Sema3B, and Sema3F are associated with increased tumor progression and decreased survival of patients. Because of the clinical relevance of semaphorin expression in breast cancer, these genes might be used as a prognostic factor for the prediction of breast cancer progression. Thus, targeting semaphorins, their receptors, and downstream signaling molecules with therapeutic drugs might be useful in inhibiting angiogenesis and metastasis for the management of breast cancer.

\section{Acknowledgment}

We thank Anuradha Bulbule for critically reading the manuscript.

\section{Disclosure}

The authors report no conflicts of interest in this work.

\section{References}

1. Siegel RL, Miller KD, Jemal A. Cancer statistics, 2016. CA Cancer J Clin. 2016;66(1):7-30.

2. Nwabo Kamdje AH, Seke Etet PF, Vecchio L, Muller JM, Krampera M, Lukong KE. Signaling pathways in breast cancer: therapeutic targeting of the microenvironment. Cell Signal. 2014;26(12):2843-2856.

3. Baskin Y, Yigitbasi T. Clinical proteomics of breast cancer. Curr Genomics. 2010;11(7):528-536.

4. Sørlie T, Perou CM, Tibshirani R, et al. Gene expression patterns of breast carcinomas distinguish tumor subclasses with clinical implications. Proc Natl Acad Sci USA. 2001;98(19):10869-10874.

5. Staton CA, Shaw LA, Valluru M, et al. Expression of class 3 semaphorins and their receptors in human breast neoplasia. Histopathology. 2011;59(2):274-282.

6. Malik MF, Satherley LK, Davies EL, Ye L, Jiang WG. Expression of semaphorin $3 \mathrm{C}$ in breast cancer and its impact on adhesion and invasion of breast cancer cells. Anticancer Res. 2016;36(3):1281-1286.

7. Kolodkin AL, Matthes DJ, Goodman CS. The semaphorin genes encode a family of transmembrane and secreted growth cone guidance molecules. Cell. 1993;75(7):1389-1399. 
8. Capparuccia L, Tamagnone L. Semaphorin signaling in cancer cells and in cells of the tumor microenvironment-two sides of a coin. $J$ Cell Sci. 2009;122(11):1723-1736.

9. Neufeld G, Kessler O. The semaphorins: versatile regulators of tumour progression and tumour angiogenesis. Nat Rev Cancer. 2008;8(8):632-645.

10. Mishra R, Kumar D, Tomar D, Chakraborty G, Kumar S, Kundu GC. The potential of class 3 semaphorins as both targets and therapeutics in cancer. Expert Opin Ther Targets. 2015;19(3):427-442.

11. Mishra R, Thorat D, Soundararajan G, et al. Semaphorin $3 A$ upregulates FOXO 3a-dependent MelCAM expression leading to attenuation of breast tumor growth and angiogenesis. Oncogene. 2015;34(12):1584-1595.

12. Appleton BA, Wu P, Maloney J, et al. Structural studies of neuropilin/ antibody complexes provide insights into semaphorin and VEGF binding. EMBO J. 2007;26(23):4902-4912.

13. Parker MW, Linkugel AD, Vander Kooi CW. Effect of C-terminal sequence on competitive semaphorin binding to neuropilin-1. $\mathrm{J} \mathrm{Mol}$ Biol. 2013;425(22):4405-4414.

14. Castro-Rivera E, Ran S, Thorpe P, Minna JD. Semaphorin 3B (SEMA3B) induces apoptosis in lung and breast cancer, whereas VEGF165 antagonizes this effect. Proc Natl Acad Sci USA. 2004;101(31):11432-11437.

15. Allegra M, Zaragkoulias A, Vorgia E, et al. Semaphorin-7a reverses the ERF-induced inhibition of EMT in Ras-dependent mouse mammary epithelial cells. Mol Biol Cell. 2012;23(19):3873-3881.

16. Cole-Healy Z, Vergani P, Hunter K, Brown NJ, Reed MW, Staton $\mathrm{CA}$. The relationship between semaphorin $3 \mathrm{C}$ and microvessel density in the progression of breast and oral neoplasia. Exp Mol Pathol. 2015;99(1):19-24

17. Bender RJ, Mac Gabhann F. Expression of VEGF and semaphorin genes define subgroups of triple negative breast cancer. PloS One. 2013;8(5):e61788.

18. Malik MF, Ye L, Jiang WG. Reduced expression of semaphorin 4D and plexin-B in breast cancer is associated with poorer prognosis and the potential linkage with oestrogen receptor. Oncol Rep. 2015;34(2):1049-1057.

19. Goodman CS, Kolodkin AL, Luo Y, Püschel AW, Raper JA. Unified nomenclature for the semaphorins/collapsins. Cell. 1999; 97(5):551-552.

20. Gherardi E, Love CA, Esnouf RM, Jones EY. The sema domain. Curr Opin Struct Biol. 2004;14(6):669-678.

21. Koppel AM, Feiner L, Kobayashi H, Raper JA. A 70 amino acid region within the semaphorin domain activates specific cellular response of semaphorin family members. Neuron. 1997;19(3):531-537.

22. Takahashi T, Strittmatter SM. Plexinal autoinhibition by the plexin sema domain. Neuron. 2001;29(2):429-439.

23. Kong-Beltran M, Stamos J, Wickramasinghe D. The Sema domain of Met is necessary for receptor dimerization and activation. Cancer Cell. 2004;6(1):75-84.

24. Tamagnone L. Emerging role of semaphorins as major regulatory signals and potential therapeutic targets in cancer. Cancer Cell. 2012;22(2):145-152.

25. Chang K, Karnad A, Zhao S, Freeman JW. Roles of c-Met and RON kinases in tumor progression and their potential as therapeutic targets. Oncotarget. 2015;(6):3507-3518.

26. Bork P, Doerks T, Springer TA, Snel B. Domains in plexins: links to integrins and transcription factors. Trends Biochem Sci. 1999;24(7):261-263.

27. Basile JR, Holmbeck K, Bugge TH, Gutkind JS. MT1-MMP controls tumor-induced angiogenesis through the release of semaphorin 4D. $J$ Biol Chem. 2007;282(9):6899-6905.

28. Messina A, Giacobini P. Semaphorin signaling in the development and function of the gonadotropin hormone-releasing hormone system. Front Endocrinol. 2013;4:133.

29. He Z, Tessier-Lavigne M. Neuropilin is a receptor for the axonal chemorepellent Semaphorin III. Cell. 1997;90(4):739-751.

30. Tamagnone L, Artigiani S, Chen H, et al. Plexins are a large family of receptors for transmembrane, secreted, and GPI-anchored semaphorins in vertebrates. Cell. 1999;99(1):71-80.
31. Janssen BJ, Robinson RA, Pérez-Brangulí F, et al. Structural basis of semaphorin-plexin signalling. Nature. 2010;467(7319):1118-1122.

32. Oinuma I, Ishikawa Y, Katoh H, Negishi M. The Semaphorin 4D receptor Plexin-B1 is a GTPase activating protein for R-Ras. Science. 2004;305(5685):862-865.

33. Kolodkin AL, Ginty DD. Steering clear of semaphorins: neuropilins sound the retreat. Neuron. 1997;19(6):1159-1162.

34. Soker S, Takashima S, Miao HQ, Neufeld G, Klagsbrun M. Neuropilin-1 is expressed by endothelial and tumor cells as an isoform-specific receptor for vascular endothelial growth factor. Cell. 1998;92(6):735-745.

35. Pellet-Many C, Frankel P, Jia H, Zachary I. Neuropilins: structure, function and role in disease. Biochem J. 2008;411(2):211-226.

36. Yazdani U, Terman JR. The semaphorins. Genome Biol. 2006;7(3):211.

37. Takahashi T, Fournier A, Nakamura F, et al. Plexin-neuropilin-1 complexes form functional semaphorin-3A receptors. Cell. 1999;99(1):59-69.

38. Casazza A, Finisguerra V, Capparuccia L, et al. Sema3E-Plexin D1 signaling drives human cancer cell invasiveness and metastatic spreading in mice. J Clin Invest. 2010;120(8):2684-2698.

39. Masuda K, Furuyama T, Takahara M, Fujioka S, Kurinami H, Inagaki S. Sema4D stimulates axonal outgrowth of embryonic DRG sensory neurones. Genes Cells. 2004;9(9):821-829.

40. Deng S, Hirschberg A, Worzfeld T, et al. Plexin-B2, but not Plexin-B1, critically modulates neuronal migration and patterning of the developing nervous system in vivo. J Neurosci. 2007;27(23):6333-6347.

41. Artigiani S, Conrotto P, Fazzari P, et al. Plexin-B3 is a functional receptor for semaphorin 5A. EMBO Rep. 2004;5(7):710-714.

42. Pan H, Wanami LS, Dissanayake TR, Bachelder RE. Autocrine semaphorin3A stimulates alpha2 beta1 integrin expression/function in breast tumor cells. Breast Cancer Res Treat. 2009;118(1):197-205.

43. Gehler S, Compere FV, Miller AM. Semaphorin 3A increases FAK phosphorylation at focal adhesions to modulate MDA-MB-231 cell migration and spreading on different substratum concentrations. Int $J$ Breast Cancer. 2017;2017:9619734.

44. Guttmann-Raviv N, Shraga-Heled N, Varshavsky A, GuimaraesSternberg C, Kessler O, Neufeld G. Semaphorin-3A and semaphorin-3F work together to repel endothelial cells and to inhibit their survival by induction of apoptosis. J Biol Chem. 2007;282(36):26294-26305.

45. Acevedo LM, Barillas S, Weis SM, Göthert JR, Cheresh DA. Semaphorin $3 \mathrm{~A}$ suppresses VEGF-mediated angiogenesis yet acts as a vascular permeability factor. Blood. 2008;111(5):2674-2680.

46. Shen WW, Chen WG, Liu FZ, et al. Breast cancer cells promote osteoblastic differentiation via Sema $3 \mathrm{~A}$ signaling pathway in vitro. Int $J$ Clin Exp Pathol. 2015;8(2):1584-1583.

47. Castro-Rivera E, Ran S, Brekken RA, Minna JD. Semaphorin 3B inhibits the phosphatidylinositol 3-kinase/Akt pathway through neuropilin-1 in lung and breast cancer cells. Cancer Res. 2008;68(20):8295-8303.

48. Shahi P, Wang CY, Chou J, et al. GATA3 targets semaphorin 3B in mammary epithelial cells to suppress breast cancer progression and metastasis. Oncogene. 2017;36(40):5567-5575.

49. Zhu X, Zhang X, Ye Z, et al. Silencing of semaphorin 3C suppresses cell proliferation and migration in MCF-7 breast cancer cells. Oncol Lett. 2017;14(5):5913-5917.

50. Mumblat Y, Kessler O, Ilan N, Neufeld G. Full-length semaphorin-3C is an inhibitor of tumor lymphangiogenesis and metastasis. Cancer Res. 2015;75(11):2177-2186.

51. Esselens C, Malapeira J, Colomé N, et al. The cleavage of semaphorin 3C induced by ADAMTS1 promotes cell migration. J Biol Chem. 2010;285(4):2463-2473.

52. Xu X, Zhao Z, Guo S, et al. Increased semaphorin 3c expression promotes tumor growth and metastasis in pancreatic ductal adenocarcinoma by activating the ERK1/2 signaling pathway. Cancer Lett. 2017;397:12-22.

53. Luchino J, Hocine M, Amoureux MC, et al. Semaphorin 3E suppresses tumor cell death triggered by the plexin D1 dependence receptor in metastatic breast cancers. Cancer Cell. 2013;24(5):673-685.

54. Wei JC, Yang J, Liu D, et al. Tumor-associated lymphatic endothelial cells promote lymphatic metastasis by highly expressing and secreting SEMA4C. Clin Cancer Res. 2017;23(1):214-224. 
55. Yang YH, Buhamrah A, Schneider A, et al. Semaphorin 4d promotes skeletal metastasis in breast cancer. PLoS One. 2016;11(2):e0150151.

56. Swiercz JM, Worzfeld T, Offermanns S. ErbB-2 and met reciprocally regulate cellular signaling via plexin-B1. J Biol Chem. 2008;283(4):1893-1901.

57. Ch'ng ES, Kumanogoh A. Roles of Sema4D and Plexin-B1 in tumor progression. Mol Cancer. 2010;9(1):251.

58. Worzfeld T, Swiercz JM, Looso M, Straub BK, Sivaraj KK, Offermanns $\mathrm{S}$. ErbB-2 signals through Plexin-B1 to promote breast cancer metastasis. J Clin Invest. 2012;122(4):1296-1305.

59. Black SA, Nelson AC, Gurule NJ, Futscher BW, Lyons TR. Semaphorin $7 \mathrm{a}$ exerts pleiotropic effects to promote breast tumor progression. Oncogene. 2016;35(39):5170-5178.

60. Garcia-Areas R, Libreros S, Amat S, et al. Semaphorin7A promotes tumor growth and exerts a pro-angiogenic effect in macrophages of mammary tumor-bearing mice. Front Physiol. 2014;5:17.

61. Schneider BP, Miller KD. Angiogenesis of breast cancer. J Clin Oncol. 2005;23(8):1782-1790.

62. Conley SJ, Gheordunescu E, Kakarala P, et al. Antiangiogenic agents increase breast cancer stem cells via the generation of tumor hypoxia. Proc Natl Acad Sci USA. 2012;109(8):2784-2789.

63. Liu TJ, Sun BC, Zhao XL, et al. CD133+ cells with cancer stem cell characteristics associates with vasculogenic mimicry in triple-negative breast cancer. Oncogene. 2013;32(5):544-553.

64. Casazza A, Fu X, Johansson I, et al. Systemic and targeted delivery of semaphorin $3 \mathrm{~A}$ inhibits tumor angiogenesis and progression in mouse tumor models. Arterioscler Thromb Vasc Biol. 2011;31(4):741-749.

65. Jiang H, Chen C, Sun Q, et al. The role of semaphorin 4D in tumor development and angiogenesis in human breast cancer. Onco Targets Ther. 2016;9:5737-5750.

66. Sierra JR, Corso S, Caione L, et al. Tumor angiogenesis and progression are enhanced by Sema4D produced by tumor-associated macrophages. $J$ Exp Med. 2008;205(7):1673-1685.

67. Seyfried TN, Huysentruyt LC. On the origin of cancer metastasis. Crit Rev Oncog. 2013;18(1-2):43-73.

68. Singh A, Settleman JE. EMT, cancer stem cells and drug resistance: an emerging axis of evil in the war on cancer. Oncogene. 2010;29(34):4741-4751.
69. Xu Z, Chen WG, Sun J, Chen P, Chu TW. Hypoxia inhibits the expression of semaphorin $3 \mathrm{~A}$ in breast cancer cells and regulates the differentiation of preosteoblast cells. Tumor. 2016;36(12):1298-1306.

70. Nasarre P, Constantin B, Rouhaud L, et al. Semaphorin SEMA3F and VEGF have opposing effects on cell attachment and spreading. Neoplasia. 2003;5(1):83-92.

71. Xiong G, Wang C, Evers BM, Zhou BP, Xu R. ROR $\alpha$ suppresses breast tumor invasion by inducing SEMA3F expression. Cancer Res. 2012;72(7):1728-1739.

72. Christensen C, Ambartsumian N, Gilestro G, et al. Proteolytic processing converts the repelling signal Sema3E into an inducer of invasive growth and lung metastasis. Cancer Res. 2005;65(14):6167-6177.

73. Garcia-Areas R, Libreros S, Simoes M, et al. Suppression of tumorderived Semaphorin 7A and genetic ablation of host-derived Semaphorin 7A impairs tumor progression in a murine model of advanced breast carcinoma. Int J Oncol. 2017;51(5):1395-1404.

74. Chen Y, Zhang L, Liu W, et al. Expression of semaphorin4C (Sema4C) in breast cancer, endometrial cancer and prostate cancer and its clinical significance. Chin J Clin Oncol. 2010;37(9):507-511.

75. Wu M, Han L, Shi Y, et al. Development and characterization of a novel method for the analysis of gene expression patterns in lymphatic endothelial cells derived from primary breast tissues. J Cancer Res Clin Oncol. 2010;136(6):863-872.

76. Yang Q, Wang Y, Lu X, et al. MiR-125b regulates epithelial-mesenchymal transition via targeting Sema4C in paclitaxel-resistant breast cancer cells. Oncotarget. 2015;6(5):3268-3279.

77. Evans EE, Jonason AS, Bussler H, et al. Antibody blockade of semaphorin 4D promotes immune infiltration into tumor and enhances response to other immunomodulatory therapies. Cancer Immunol Res. 2015;3(6):689-701.

78. Rody A, Holtrich U, Gaetje R, et al. Poor outcome in Estrogen receptor-positive breast cancers predicted by loss of Plexin B1. Clin Cancer Res. 2007;13(4):1115-1122.

79. Rody A, Karn T, Ruckhäberle E, et al. Loss of Plexin B1 is highly prognostic in low proliferating ER positive breast cancers-results of a large scale microarray analysis. Eur J Cancer. 2009;45(3):405-413.

80. Chen D, Li Y, Wang L, Jiao K. SEMA6D expression and patient survival in breast invasive carcinoma. Int J breast cancer. 2015;2015:539721.
Breast Cancer - Targets and Therapy

\section{Publish your work in this journal}

Breast Cancer - Targets and Therapy is an international, peerreviewed open access journal focusing on breast cancer research, identification of therapeutic targets and the optimal use of preventative and integrated treatment interventions to achieve improved outcomes, enhanced survival and quality of life for the cancer patient.

\section{Dovepress}

The manuscript management system is completely online and includes a very quick and fair peer-review system, which is all easy to use. Visit http://www.dovepress.com/testimonials.php to read real quotes from published authors. 